\title{
Textural Hausdorff Distance for Wider-Range Tolerance to Pose Variation and Misalignment in 2D Face Recognition
}

\author{
Sanqiang Zhao and Yongsheng Gao \\ School of Engineering, Griffith University, Nathan Campus \\ 170 Kessels Road, Brisbane, QLD 4111, Australia \\ \{s.zhao, yongsheng.gao\}@griffith.edu.au
}

\begin{abstract}
This paper addresses two critical but rarely concerned issues in $2 D$ face recognition: wider-range tolerance to pose variation and misalignment. We propose a new Textural Hausdorff Distance (THD), which is a compound measurement integrating both spatial and textural features. The THD is applied to a Significant Jet Point (SJP) representation of face images, where a varied number of shape-driven SJPs are detected automatically from low-level edge map with rich information content. The comparative experiments conducted on publicly available FERET and AR face databases demonstrated that the proposed approach has a considerably wider range of tolerance against both in-depth head rotation and face misalignment.
\end{abstract}

\section{Introduction}

Face recognition has attracted much attention from both academic and industrial communities. While the state of the art in face recognition has demonstrated a certain level of maturity under controlled condition [1], it is still far from adequate to deal with uncontrolled condition [2]. There have been extensive research efforts to counteract facial expressions, illumination changes and pose variations. However, little attention is received on two critical issues, wider-range tolerance to pose variation and face misalignment, in 2D face recognition.

Pose variation has been identified as one of the prominent challenges in face recognition [2]. The classical way of tackling this problem is by constructing multi-view representations. It is capable of covering large pose variations, but requires multiple sample images per person at different viewing angles. These images can be real views [3], or virtual views synthesized by incorporating prior knowledge from example faces [4] or more complicated 3D face models [5]. However, there is another way of addressing this problem through increasing the pose tolerance range of face classifiers. Existing posetolerant face recognition systems (e.g. [6, 7]) perform well only within a narrow range of rotation angles (generally $\pm 15^{\circ}$ ). Once the rotation angle becomes large, the system performance deteriorates abruptly. In fact, the importance of increasing pose tolerance ability of face classifiers has been underestimated. It not only makes a single-view face recognizer more reliable, but also can reduce the numbers of viewing angles in a multi-view face recognizer. Fewer numbers of viewing angles mean less laborious effort for collecting, storing and processing sample images.

Misalignment is an inevitable problem in face recognition. Although people have been developing automatic localization systems, aligning faces with accuracy, consistency and robustness is still an open question. The performance of face recognition approaches is severely affected by the face alignment accuracy. For example, the rank-one recognition rate of Fisherface method dropped by $10 \%$ with only 1-pixel misalignment, and dropped by $50 \%$ with 2-pixel misalignment [8]. In spite of its importance, the misalignment problem receives less attention in the research communities. One of the sporadic exceptions is Martínez's work [9], in which a probabilistic approach was employed to compensate for the localization errors. However, the performance of his approach is affected by the lighting changes. In [10], Ahonen et al. compared the recognition rates of Local Binary Pattern (LBP) method and Principal Component Analysis (PCA) method [11] with the effect of misalignment. While LBP significantly outperformed PCA for various localization errors, both LBP and PCA degraded steeply as the misalignment increased. Other researchers reported investigations of tackling the misalignment problem through intentionally perturbing the eye locations of the gallery images [8] or the probe images [12], which augment the number of the "virtual" images and considerably increase the computational efforts and the storage space.

The Hausdorff distance was originally defined as a dissimilarity measure on two point sets, and was later employed as a shape comparison metric on binary images 
[13]. Unlike most shape comparison methods that build a point-to-point correspondence between a model and a test image, the Hausdorff distance can be calculated without explicit pairing of points in their respective data sets. Considering that the original Hausdorff distance is very sensitive to outlier points, Dubuisson and Jain [14] presented a modified Hausdorff distance (MHD) to alleviate the sensitivity problem and obtained more desirable performance. Takács [15] employed a "doubly" modified Hausdorff distance (M2HD) to compare frontal face images. The M2HD method uses a penalty function to give preference to points located within a predefined neighborhood. Gao and Qi [16] integrated the structural information into the Hausdorff distance for face retrieval. The structural information is represented by direction angles extracted from neighboring points. The method is robust to illumination variations but sensitive to facial expression changes. Xie and Lam [17] employed an elastic shape-texture matching method for face recognition, in which a variant of Hausdorff distance is devised on edge maps. In stead of using edge maps, Vivek and Sudha [18] proposed a robust Hausdorff distance measure, which was applied on LBP-like patterns extracted from each pixel of an image. Because the distance measure is based on the whole images, the computational work is intensive. The approach is also sensitive to large range of pose variations.

In this paper, we propose a new Textural Hausdorff Distance (THD) to deal with the two aforementioned issues. The THD employs both spatial and textural information and is a compound measurement. It inherits the advantages of Hausdorff distance and can be calculated without explicit correspondence between respective data sets. We apply the THD measurement to a Significant Jet Point (SJP) representation of face images. An SJP is defined as a significant point attached with its Gabor coefficients (jet). Each face is represented by a set of SJPs to describe both spatial and textural information. To better represent diverse facial characteristics of different individuals, the significant points are detected from lowlevel edge map with rich information content on the face image. Both the number and the locations of significant points are varied from person to person. This is different from existing Gabor-based methods (e.g. [19-21]), in which face recognition was performed through comparing Gabor features of equal numbers of fiducial points across different individuals. The fiducial points were located either through elastic graph matching $[19,20]$ or through third-party localization algorithms [21] to establish explicit point pairings. Whichever way, the localization accuracy is a major issue that affects the recognition performance. By using the THD measurement, we can circumvent the process of conducting exhaustive search to find correspondence between significant points. The effectiveness of the proposed approach was investigated and evaluated on two publicly available FERET [1] and
AR [22] face databases. The experimental results demonstrated that the proposed approach has a considerably wider range of tolerance against both indepth head rotation and face misalignment.

The rest of the paper is organized as follows. Section 2 describes the SJP representation for face images. Section 3 introduces the original Hausdorff distance and defines the THD measurement. In Section 4, comparative experiments on FERET and AR databases are reported to evaluate the performance of the proposed approach. The last section concludes the paper with some discussions.

\section{Representation}

As the proposed Textural Hausdorff Distance measurement is applied to the Significant Jet Point (SJP) representation of face images, we give a brief description of the SJP representation in this section.

\subsection{Significant Point Detection}

The edges in an image provide strong identity descriptive capability for classification [23]. Containing spatial information, significant points are detected at positions which have rich edge information in a face image. In contrast to traditional methods where feature points are often predefined as the locations of eyes, nose, mouth, etc., we do not fix either the number or the locations of significant points. The number of significant points and their locations can vary in order to better represent diverse facial characteristics of different persons, such as dimples, moles (see the left hand image in Figure 1 ), etc. These diverse features are also important cues that humans might use for recognizing faces.

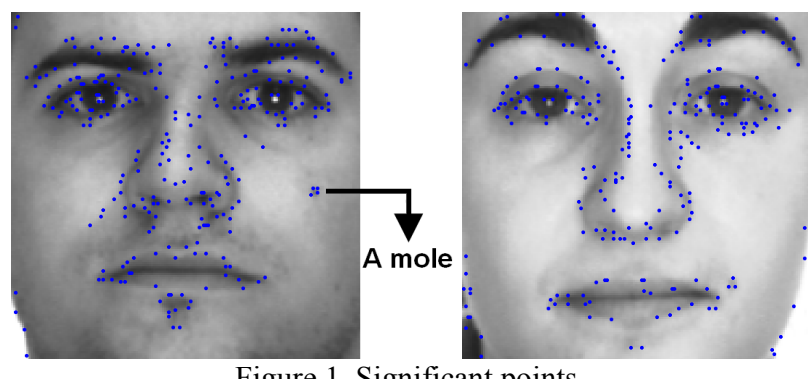

Figure 1. Significant points.

In order to ensure less demand on storage space and less sensitivity to illumination changes, the significant points should be placed on the significant edge curves with high curvatures. While any general edge detection method can be used to detect the significant points, we use an edge detector from [24], followed by the Dynamic Two-Strip algorithm [25] to obtain these points. A comparative evaluation of interest point detectors was reported in [26]. Figure 1 illustrates two examples of significant points superimposed on the face image from AR database. 


\subsection{Significant Jet Point}

The local intensity distribution around each significant point is important for face recognition. To extract the textural information, Gabor features are employed in this paper. Gabor wavelets offer desirable characteristics of capturing salient visual properties such as spatial localization and orientation selectivity, and provide the optimized resolution in both spatial and frequency domains [6]. The 2D Gabor kernel functions used for feature extraction are

$$
\psi_{j}(\mathbf{x})=\frac{k_{j}^{2}}{\sigma^{2}} \exp \left(-\frac{k_{j}^{2} x^{2}}{2 \sigma^{2}}\right)\left[\exp \left(i \mathbf{k}_{j} \mathbf{x}\right)-\exp \left(-\frac{\sigma^{2}}{2}\right)\right],
$$

where

$$
\mathbf{k}_{j}=\left(\begin{array}{l}
k_{j x} \\
k_{j y}
\end{array}\right)=\left(\begin{array}{c}
k_{v} \cos \varphi_{\mu} \\
k_{v} \sin \varphi_{\mu}
\end{array}\right), k_{v}=2^{-\frac{v+2}{2}} \pi, \quad \varphi_{\mu}=\mu \frac{\pi}{8} .
$$

The index $j=\mu+8 v$ covers a discrete set of five different frequencies $v=0, \cdots, 4$ and eight orientations $\mu=0, \cdots, 7$. The width of the Gaussian is controlled by the parameter $\sigma=2 \pi$ [19]. Extracted from one pixel $\mathbf{x}=(x, y)$ in an image $I(\mathbf{x})$, a jet $J$ is the set $\left\{J_{j}\right\}$ of 40 convolution coefficients using Gabor kernels of different frequencies and orientations:

$$
J_{j}(\mathbf{x})=\int I\left(\mathbf{x}^{\prime}\right) \psi_{j}\left(\mathbf{x}-\mathbf{x}^{\prime}\right) d^{2} \mathbf{x}^{\prime} .
$$

After significant points are detected, Gabor jet is extracted from each point. An SJP is defined as the Cartesian coordinate $\mathbf{x}=(x, y)$ of a significant point attached with a jet extracted from this point:

$$
S J P=(\mathbf{x} ; J) .
$$

Based on the SJP description, a face is represented by a set of SJPs integrating both spatial and textural cues. The SJP representation, using sparse shape-driven points, not only reduces the storage demand of an image, but also is less sensitive to illumination changes due to the fact that it is derived from low-level illumination-insensitive edge maps. Moreover, Gabor features attached on the points significantly enhance the discriminative power of the representation to improve the recognition accuracy.

\section{Measurement}

In this section, we first briefly introduce the original Hausdorff distance and its modified version, and then present the proposed Textural Hausdorff Distance (THD) measurement for the SJP representation of face images.

\subsection{Hausdorff Distance}

The original Hausdorff distance is defined as

$$
H D(A, B)=\max \left(d_{-} H D(A, B), d_{-} H D(B, A)\right),
$$

where $A=\left\{\mathbf{a}_{1}, \mathbf{a}_{2}, \cdots, \mathbf{a}_{m}\right\}$ and $B=\left\{\mathbf{b}_{1}, \mathbf{b}_{2}, \cdots, \mathbf{b}_{n}\right\}$ are two point sets. The directed Hausdorff distance $d_{-} H D(\cdot, \cdot)$ is given by

$$
d_{-} H D(A, B)=\max _{\mathbf{a} \in A} \min _{\mathbf{b} \in B} d(\mathbf{a}, \mathbf{b})=\max _{\mathbf{a} \in A} \min _{\mathbf{b} \in B}\|\mathbf{a}-\mathbf{b}\|,(6)
$$

where $\|\cdot\|$ is the norm of a vector. The directed Hausdorff distance $d_{-} H D(A, B)$ identifies the point $\mathbf{a} \in A$ whose distance from its nearest point in $B$ is maximum among all points in $A$, and measures the distance from a to its nearest point in $B$. In other words, it finds the distance between the most mismatching point in $A$ and its nearest point in $B$. Usually $d_{-} H D(A, B)$ and $d_{-} H D(B, A)$ are different. The Hausdorff distance is the maximum of $d_{-} H D(A, B)$ and $d_{-} H D(B, A)$. Therefore, it measures the degree of mismatch between two point sets by comparing the distance of the point in $A$ that has the largest distance from any point in $B$, and vice versa.

The original Hausdorff distance is very sensitive to outlier points. A few outlier points, even only a single one, can perturb the distance greatly, though the two point sets might be very similar. Dubuisson and Jain [14] employed a modified Hausdorff distance (MHD):

$$
\operatorname{MHD}(A, B)=\max \left(d \_M H D(A, B), d \_M H D(B, A)\right),(7)
$$

in which the directed MHD is defined as

$$
d_{-} \operatorname{MHD}(A, B)=\frac{1}{N_{A}} \sum_{\mathbf{a} \in A} \min _{\mathbf{b} \in B}\|\mathbf{a}-\mathbf{b}\|,
$$

where $N_{A}=m$ is the number of points in set $A$. This modified distance calculates the average distance from each point $\mathbf{a} \in A$ to its nearest point in $B$. It can alleviate the sensitivity to outlier points and has more desirable performance.

\subsection{Textural Hausdorff Distance}

The THD includes both spatial measurement and textural measurement. In an SJP, spatial information is represented by the coordinate of the significant point. For two SJPs $S J P=(\mathbf{x} ; J)$ and $S J P^{\prime}=\left(\mathbf{x}^{\prime} ; J^{\prime}\right)$, the spatial distance is measured by $d\left(\mathbf{x}, \mathbf{x}^{\prime}\right)=\left\|\mathbf{x}-\mathbf{x}^{\prime}\right\|$. The textural information of an SJP is represented by the Gabor jet extracted from the significant point. As a set of complex numbers, a jet can be written as the polar form $J_{j}=a_{j} \exp \left(i \phi_{j}\right)$, where jet magnitudes $a_{j}(\mathbf{x})$ vary slowly with spatial position and jet phases $\phi_{j}(\mathbf{x})$ rotate with a rate set by the spatial frequency or wave vector $\mathbf{k}_{j}$ of the kernels [19]. We use a magnitude similarity function to measure two jets $J$ and $J^{\prime}$ as 


$$
S\left(J, J^{\prime}\right)=\frac{\sum_{j} a_{j} a_{j}^{\prime}}{\sqrt{\sum_{j} a_{j}^{2} \sum_{j} a_{j}^{\prime 2}}},
$$

because it provides certain robustness to position variation.

Given two finite SJP sets $M=\left\{S J P_{1}^{M}, S J P_{2}^{M}, \cdots, S J P_{P}^{M}\right\}$ representing a model face in the database and $T=\left\{S J P_{1}^{T}, S J P_{2}^{T}, \cdots, S J P_{Q}^{T}\right\}$ representing a test face from input, where $P$ and $Q$ are the numbers of SJPs in $M$ and $T$ respectively. The THD is defined as

$T H D(M, T)=\max \left(d \_T H D(M, T), d \_T H D(T, M)\right),(10)$ where the directed THD function $d_{-} \operatorname{THD}(M, T)$ is defined as

$$
d_{-} T H D(M, T)=\frac{1}{P} \sum_{1 \leq p \leq P} \min _{1 \leq q \leq Q}\left(d\left(\mathbf{x}_{p}^{M}, \mathbf{x}_{q}^{T}\right)+\lambda\left(1-S\left(J_{p}^{M}, J_{q}^{T}\right)\right)\right) .
$$

This directed THD function calculates the average distance from each $\operatorname{SJP}_{p}^{M}(1 \leq p \leq P)$ to its nearest neighbor in $T$. The distance between two SJPs is measured through integrating spatial distance of significant points and magnitude similarity of Gabor jets. The THD is a compound measurement composed of both spatial and textural information. The weight $\lambda$ is used to balance the contributions of spatial distance and jet dissimilarity. For a test image, the face recognizer calculates the THD between the test face and each model face in the database. The model with minimum distance is considered as the correct match.

\section{Experiments}

To validate the proposed THD approach, we conducted comparative experiments on two publicly available FERET [1] and AR [22] face databases. The two databases were employed to evaluate the tolerance ability of the proposed approach against pose variation and misalignment respectively. In all the experiments, the proposed approach was compared with four benchmark methods: 1) Principal Component Analysis (PCA) method [11], which is a widely used baseline algorithm in appearance-based face recognition; 2) Modified Hausdorff distance (MHD) method [14], which could be considered as a baseline algorithm in Hausdorff-based face recognition; 3) Structural Hausdorff distance method [16]; and 4) Local Binary Pattern (LBP) method, which is one of the state-of-the-art algorithms and has proved to outperform PCA, Bayesian and EBGM methods [10].

\subsection{Database}

The FERET database is one of the standard databases to evaluate face recognition algorithms. The pose subset of the database was used in our experiments. This subset includes 1400 images of 200 persons (each person has 7 images). The images were categorized and named with two-character strings as "ba", "bc", "bd", "be", "bf", "bg" and "bh". These strings indicate different angles of indepth head rotation in the horizontal direction (ranging from $+40^{\circ}$ to $-40^{\circ}$ ). Each image was normalized (in scale and in-plane rotation) and cropped to $120 \times 160$ pixels based on the locations of two eyes.

The AR database contains 120 complete sets of images from 120 persons ( 65 men and 55 women). Each person has 26 images, recorded in two different sessions with a two-week time interval. These images cover frontal view faces under controlled condition, different facial expressions and different illumination conditions. All the images were normalized and cropped to $160 \times 160$ pixels.

\subsection{Parameter Tuning}

In Equation (11), the weight $\lambda$ balances the contributions of spatial and textural measurements. To determine $\lambda$, a set of experiments with different values of $\lambda$ was conducted on the AR database. The neutral expression faces in the first session were used to construct the gallery set, and the neutral faces in the second session were selected as the probe set. The rank-one recognition rate against the weight $\lambda$ is plotted in Figure 2 . The recognition rate reached and remained maximum when $\lambda$ ranged from 100 to 1000 . The weight $\lambda=160$ was selected and used in the rest of the experiments.

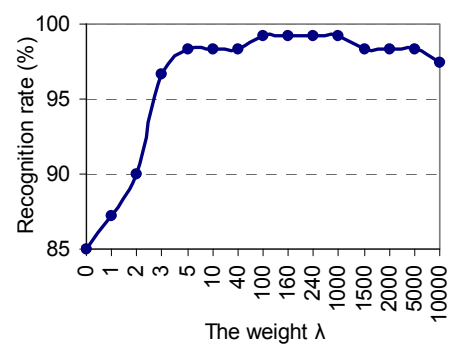

Figure 2. Rank-one recognition rate against the weight $\lambda$ on the AR database.

\subsection{Tolerance Analysis on Pose Variation}

The FERET database was used to evaluate the effect of pose variation on the performance of the proposed THD approach. The "ba" category (frontal faces) was used as the gallery set, and the remaining categories were used as the probe sets respectively. The comparative rank-one recognition rates as a function of rotation angles are plotted in Figure 3. It was observed that THD and LBP performed consistently better than structural Hausdorff distance, PCA and MHD methods for all the poses. With small rotation angles $\left( \pm 15^{\circ}\right)$, the average recognition rate 
of THD was $4.8 \%$ higher than that of LBP, but over $25.8 \%$ higher than those of the other three methods. As the rotation angle increased, the recognition accuracy of THD dropped much slower than that of all benchmark methods. The average performance drop of THD from $\pm 15^{\circ}$ to $\pm 25^{\circ}$ was only $7.8 \%$, whereas the drops of LBP, structural Hausdorff distance, PCA and MHD were 31.4\%, 44.1\%, $41.3 \%$ and $54 \%$, respectively. At the rotation angles of $\pm 25^{\circ}$, THD accomplished an average recognition rate of $86 \%$, while the average rates of benchmark methods were all below $61 \%$. Overall, THD maintained a flat recognition performance within a wider range of rotation angles at least up to $\pm 25^{\circ}$. When the rotation angle reached to $\pm 40^{\circ}$, the average recognition rate of THD became unsatisfactory, although higher than those of benchmark methods. These experimental results demonstrated widerrange tolerance of the proposed approach against in-depth head rotation.

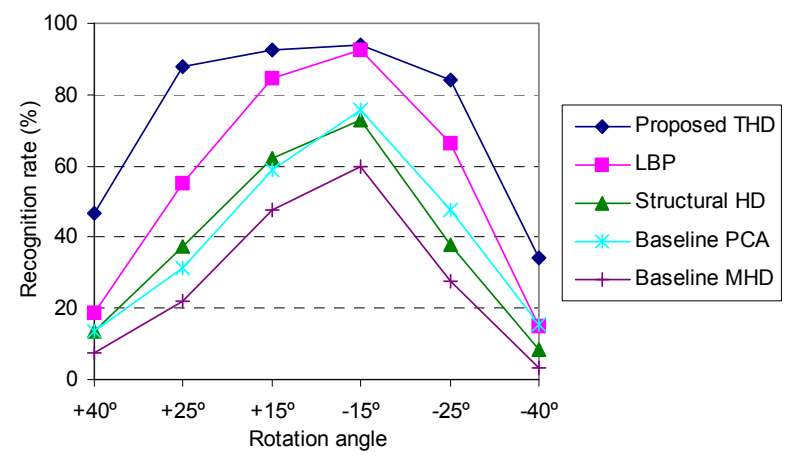

Figure 3. Comparative rank-one recognition rates against pose variation on the FERET database.

\subsection{Tolerance Analysis on Misalignment}

The AR database was used to evaluate the effect of misalignment on the recognition rate of the proposed approach. All the neutral faces in the first session were used as the gallery images. The neutral faces in the second session were used as the probe images. Before the probe images were normalized, two random vectors $\left(\Delta X_{1}, \Delta Y_{1}\right)$ and $\left(\Delta X_{2}, \Delta Y_{2}\right)$ were added to the coordinates of the left and right eyes, respectively. These "misaligned" coordinates, instead of accurate eye locations, were used to normalize the probe images. $\Delta X_{1}, \Delta Y_{1}, \Delta X_{2}$ and $\Delta Y_{2}$ were uncorrelated and normally distributed with zero mean and a standard deviation of $\sigma$. The $\sigma$ varied from 0 to 20 with an interval of 2.5. The comparative rank-one recognition rates as a function of $\sigma$ are illustrated in Figure 4, showing that THD and LBP maintained higher recognition accuracy over other three methods (structural Hausdorff distance, MHD and PCA). With a small range of misalignment $(\sigma \leq 2.5)$, THD obtained almost same performance as LBP, demonstrating that both THD and
LBP have good discriminative ability for near-frontal images. When $\sigma$ increased further to 10 , the recognition rate of THD decreased from $97.5 \%$ to $75 \%$. The performance of LBP, however, dropped significantly from $98.3 \%$ to $30.8 \%$. The performance deterioration of THD was only one third of that of LBP. The performance of structural Hausdorff distance, MHD and PCA even reduced down to $27.5 \%, 20.8 \%$ and $16.7 \%$ respectively. These experimental results demonstrated wider-range tolerance of THD over the benchmark methods in terms of face misalignment. The wider-range tolerance of the proposed method relaxes the strict demand for accurate face localization, which is not always available in practice, but often required in most existing $2 \mathrm{D}$ face recognition systems.

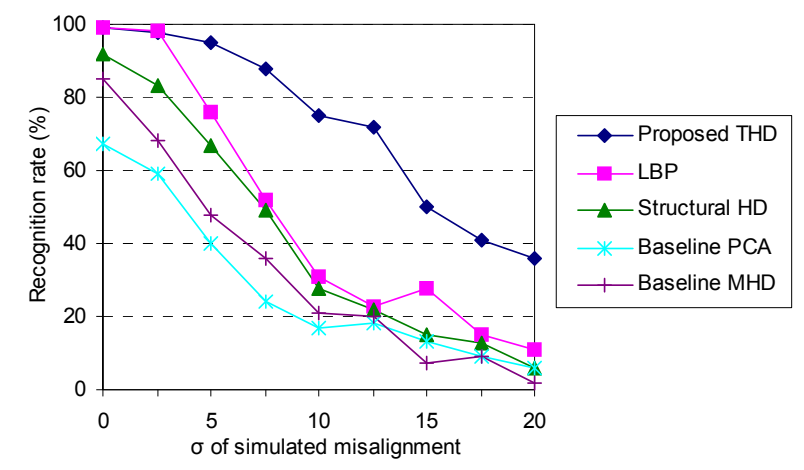

Figure 4. Comparative rank-one recognition rates against misalignments on the AR database.

In all experiments, we implemented an LBP system in which 8 points in $3 \times 3$ neighborhood are sampled; each image is partitioned into $10 \times 10$-sized windows; and equally quantized 32 histogram bins are accumulated in each window. As for the PCA system, the first 40 principal components were employed in our experiments.

\section{Conclusion and Discussion}

Wider-range tolerance to pose variation and misalignment are two important but less concerned issues in $2 \mathrm{D}$ face recognition. They cause abrupt performance degradation in many automatic systems. Both pose variation and misalignment break down the pixel-level correspondence between face images, which is an underlying assumption in many appearance based face recognition approaches. This paper, for the first time, aims specifically at these two issues and proposes a new textural Hausdorff distance for face recognition. The proposed measurement integrates both spatial and textural information to distinguish faces. It circumvents traditional local searching strategy and is more reliable. Comparative experiments demonstrated that the proposed approach had a considerably wider range of tolerance against both head in-depth rotation and face misalignment. On the FERET 
database, it achieved an average recognition rate of $86 \%$ with rotation angles of $\pm 25^{\circ}$; and on the AR database, it obtained $75 \%$ recognition accuracy with large scope of misalignment $(\sigma=10)$. These experimental results are much better than those of the benchmark methods and also, to our knowledge, are the best reported results in 2D face recognition.

The noticeable performance of the proposed approach comes from several aspects. First of all, the identityoriented significant points contain discriminative features such as prominent irregularities in facial skin. This is different from conventional recognition algorithms, where holistic methods (e.g. Eigenface) treat local variations as noise, and analytic methods (e.g. EBGM) fail to explicitly represent these valuable individual distinctions. Another aspect is the cooperation of Gabor features and Hausdorff distance matching strategy. Gabor jets have proved to have certain robustness against small-range pose variation when explicit jet correspondences could be established. However, we demonstrated that Gabor jets could also be used through SJP representation and Hausdorff matching concept to achieve a wider range of tolerance against pose variation and misalignment. By using the Hausdorff matching strategy we could avoid jet based localization which is computationally intensive and difficult to be optimized.

\section{Acknowledgments}

This research was supported by the Australian Research Council (ARC) Discovery Grants DP0451091 and DP0877929.

\section{References}

[1] P.J. Phillips, H. Moon, S.A. Rizvi, and P.J. Rauss, "The FERET Evaluation Methodology for Face-Recognition Algorithms," IEEE Trans. PAMI, 22(10): 1090-1104, 2000.

[2] W. Zhao, R. Chellappa, P.J. Phillips, and A. Rosenfeld, "Face Recognition: A Literature Survey," ACM Computing Surveys, 35(4): 399-459, 2003.

[3] D.J. Beymer, "Face Recognition under Varying Pose," Proc. Int'l Conf. Computer Vision and Pattern Recognition, pp. 756-761, 1994.

[4] D. Beymer and T. Poggio, "Face Recognition from One Example View," Proc. Int'l Conf. Computer Vision, pp. 500507, 1995.

[5] V. Blanz and T. Vetter, "Face Recognition Based on Fitting a 3D Morphable Model," IEEE Trans. PAMI, 25(9): 10631074, 2003.

[6] M. Lades, J.C. Vorbrüggen, J. Buhmann, J. Lange, C. von der Malsburg, R.P. Würtz, and W. Konen, "Distortion Invariant Object Recognition in the Dynamic Link Architecture," IEEE Trans. Computers, 42(3): 300-311, 1993.

[7] O. de Vel and S. Aeberhard, "Line-Based Face Recognition under Varying Pose," IEEE Trans. PAMI, 21(10): 10811088, 1999.
[8] S. Shan, Y. Chang, W. Gao, B. Cao, and P. Yang, "Curse of Mis-alignment in Face Recognition: Problem and a Novel Mis-alignment Learning Solution," Proc. Int'l Conf. Automatic Face and Gesture Recognition, pp. 314-320, 2004.

[9] A.M. Martínez, "Recognizing Imprecisely Localized, Partially Occluded, and Expression Variant Faces from a Single Sample per Class," IEEE Trans. PAMI, 24(6): 748763, 2002.

[10] T. Ahonen, A. Hadid, and M. Pietikäinen, "Face Description with Local Binary Patterns: Application to Face Recognition," IEEE Trans. PAMI, 28(12): 2037-2041, 2006.

[11] M. Turk and A. Pentland, "Eigenfaces for Recognition," $J$. Cognitive Neuroscience, 3(1): 71-86, 1991.

[12] J. Min, K.W. Bowyer, and P.J. Flynn, "Eye Perturbation Approach for Robust Recognition of Inaccurately Aligned Faces," Proc. Int'l Conf. Audio- and Video-Based Biometric Person Authentication, pp. 41-50, 2005.

[13] D.P. Huttenlocher, G.A. Klanderman, and W.J. Rucklidge, "Comparing Images Using the Hausdorff Distance," IEEE Trans. PAMI, 15(9): 850-863, 1993.

[14] M.P. Dubuisson and A.K. Jain, "A Modified Hausdorff Distance for Object Matching," Proc. Int'l Conf. Pattern Recognition, pp. 566-568, 1994.

[15] B. Takács, "Comparing Face Images Using the Modified Hausdorff Distance," Pattern Recognition, 31(12): 18731881, 1998.

[16] Y. Gao and Y. Qi, "Robust Visual Similarity Retrieval in Single Model Face Databases," Pattern Recognition, 38(7): 1009-1020, 2005.

[17] X. Xie and K.M. Lam, "Elastic Shape-Texture Matching for Human Face Recognition," Pattern Recognition, 41(1): 396405, 2008.

[18] E.P. Vivek and N. Sudha, "Robust Hausdorff Distance Measure for Face Recognition," Pattern Recognition, 40(2): 431-442, 2007.

[19] L. Wiskott, J.M. Fellous, N. Krüger, and C. von der Malsburg, "Face Recognition by Elastic Bunch Graph Matching," IEEE Trans. PAMI, 19(7): 775-779, 1997.

[20] B. Duc, S. Fischer, and J. Bigün, "Face Authentication with Gabor Information on Deformable Graphs," IEEE Trans. Image Processing, 8(4): 504-516, 1999.

[21] D. González-Jiménez and J.L. Alba-Castro, "Shape-Driven Gabor Jets for Face Description and Authentication," IEEE Trans. Information Forensics and Security, 2(4): 769-780, 2007.

[22] A.M. Martínez and R. Benavente, "The AR Face Database," CVC Technical Report \#24, June 1998.

[23] Y. Gao and M.K.H. Leung, "Face Recognition Using Line Edge Map," IEEE Trans. PAMI, 24(6): 764-779, 2002.

[24] R. Nevatia and K.R. Babu, "Linear Feature Extraction and Description," Computer Graphics and Image Processing, 13(3): 257-269, 1980.

[25] M.K. Leung and Y.-H. Yang, "Dynamic Two-Strip Algorithm in Curve Fitting," Pattern Recognition, 23(1-2): 69-79, 1990.

[26] C. Schmid, R. Mohr, and C. Bauckhage, "Evaluation of Interest Point Detectors," Int'l J. Computer Vision, 37(2): 151-172, 2000. 